\title{
The nature of the absorber in the INTEGRAL highly obscured sgHMXB IGR J17252-3616
}

\author{
Antonios Manousakis* \\ ISDC Data Center for Astrophysics, Switzerland \\ E-mail: Antonios. Manousakis@unige.ch \\ Roland Walter \\ ISDC Data Center for Astrophysics, Switzerland
}

\begin{abstract}
INTEGRAL played a key role in discovering obscured sgHMXB in the Galaxy. We used XMMNewton to perform X-ray wind tomography of a specific of these systems, IGR J17252-3616, featuring eclipses of the accreting pulsar. The X-ray band $(0.2-10 \mathrm{keV})$ reveals vital information on the geometry of the surrounding gas probing simultaneously the absorption and the fluorescence emission. The XMM observations were scheduled to cover as many orbital phases as possible. Timing analysis allows the derivation of an accurate orbital solution and of the system parameters. Spectral analysis revealed remarkable variations of the absorbing column density along the orbit and of the $\mathrm{Fe} \mathrm{K} \alpha$ fluorescence line around the eclipse. The combination of these observables revealed a highly asymmetric and unprecedentedly extended structure in the stellar wind extending up to 2-3 stellar radii. The observations can be modeled in terms of three independent components:

i) the unperturbed stellar wind

ii) the contribution of a highly asymmetric hydrodynamic wind tail-like structure and

iii) a cusp of material close to the neutron star.
\end{abstract}

These dynamical structures are imaged for the first time in a sgHMXB and explain the source of the high obscuration.

8th INTEGRAL Workshop 'The Restless Gamma-ray Universe'

September 27-30 2010

Dublin Castle, Dublin, Ireland

* Speaker. 


\section{Introduction}

High mass X-ray binaries (HMXB) consist of a neutron star or a black hole fueled by the accretion of the wind of an early-type stellar companion. Their X-ray emission, a measure of the accretion rate, shows a variety of transient to persistent patterns. Outbursts are observed on timescales from seconds to months and dynamical ranges varying by factors of $10^{4}$. The majority of the known HMXB are Be/X-ray binaries, with Be stellar companions. These systems are transient, featuring bright outbursts with typical durations on the order of several weeks [16]. A second class of HMXBs harbor OB supergiant companions (sgHMXBs) that feed the compact object by means of strong, radiatively driven stellar winds or Roche lobe overflow. Thanks to INTEGRAL, the number of known sgHMXB systems has tripled in the past few years [13].

IGR J17252 - 3616 was detected by ISGRI onboard INTEGRAL on February 9, 2004 among other hard X-ray sources [12]. The source was first detected by EXOSAT (EXO 1722-3616) as a weak soft X-ray source, back in 1984 [15]. In 1987, Ginga performed a pointed observations and revealed a highly variable X-ray source, X1722-363, with a pulsation period of $\sim 413.9 \mathrm{sec}$ [10]. Additional Ginga observations revealed the orbital period of 9-10 days and a mass of the companion star of $\sim 15 M_{\odot}$ [9]. Both studies concluded that the system is a high mass X-ray binary (HMXB).

INTEGRAL and XMM - Newton observations of IGR J17252 - 3616 allowed Zurita-Heras et al. (2006) to identify the infrared counterpart of the system, to accurately measure the absorbing column density, and refine the spin period of the system [17]. Thanks to the eclipses, an accurate orbital period could be derived from INTEGRAL data. Further RXTE observations helped identify a highly inclined system $\left(i>61^{\circ}\right)$ with a companion star of $\mathrm{M}_{*} \lesssim 20 \mathrm{M}_{\odot}$ and $\mathrm{R}_{*} \sim 20-40 \mathrm{R}_{\odot}[11$, 4]. Recent VLT observations help to infer the companion spectral type (between B0-B5 I and B0B1 Ia) and radial velocity measurements [6]. Its spectral energy distribution can be characterized by a temperature of $\mathrm{T}_{*} \sim 30 \mathrm{kK}$ and a reddening of $\mathrm{A}_{V} \sim 20$ [8].

\section{Results}

The Science Analysis Software (XMM-SAS) version 9.0.0 ${ }^{1}$ was used to produce event lists for the EPIC-pn instrument. Spectra and lightcurves were built by collecting double and single events in the energy range $0.2-10 \mathrm{keV}$. The observations were scheduled to cover the orbital phases, 0.01 , $0.03,0.08,0.15,0.27,0.37,0.40,0.65,0.79$, and 0.91 inferred from the orbital solutions.

\section{Orbital solution}

We have obtained a refined orbital solution based on RXTE [11] and XMM-Newton Pulse Arrival Times [5] to derive accurate orbital phases. The orbital period is constrained using INTEGRAL $^{2}$ long-term monitoring. We have obtained an orbital period of $P=9.742 \pm 0.001$ days, a projected semi-major axis $\alpha_{\chi} \sin i=102 \pm 8$ lt-s, a mid-eclipse $T_{90}=M J D 53761.67 \pm 0.1$ and an $90 \%$ upper limit $e<0.15$ for eccentricity, yielding a mass function $f \sim 11.7 \mathrm{M}_{\odot}$. Corresponding

\footnotetext{
${ }^{1} \mathrm{http} / / / \mathrm{xmm} . \mathrm{esac}$. esa.int/sas/

${ }^{2}$ HEAVENS [14] interface available at http: //www. isdc. unige.ch/heavens
} 
masses of both donor star and compact object are in the range, $M_{O B} \approx 14-17 \mathrm{M}_{\odot}$ and $M_{N S} \approx 1.4$ $-1.7 \mathrm{M}_{\odot}$, respectively.

\section{Spectral Variability}

The spectra are always heavily absorbed below $\sim 3 \mathrm{keV}$ and contain an iron K-edge at 7.2 $\mathrm{keV}$. Throughout our observations an iron $\mathrm{K} \alpha$ line, at $6.40 \pm 0.03 \mathrm{keV}$, is always present. Some parameters (photon index, cut-off energy, blackbody templrature) did not vary significantly among the observations. To search for spectral variability we decided to fix them to their averages values $\left(\mathrm{E}_{C}=8.2 \mathrm{keV}, \Gamma=0.02, \mathrm{kT}_{B B}=0.5 \mathrm{keV}\right)$. The model was built by using an intrinsically absorbed high energy cut-off power-law with a gaussian line, a black-body component responsible for the observed soft X-ray excess, and an overall absorption responsible for the interstellar absorption fixed to the Galactic absorption $\mathrm{N}_{H}=1.5 \times 10^{22} \mathrm{~cm}^{-2}$. The model can be described as wabs* (bbody + gauss + vphabs*cutoff) in XSPEC ${ }^{3}$ using standard abundances obtained from [1]. All the normalization parameters and the intrinsic column density were free to vary. Figure 1 shows the variations of intrinsic $\mathrm{N}_{H}$ (left) and the iron $\mathrm{K} \alpha$ line corrected equivalent width $^{4}$ (right) as a function of phase together with a wind model described in the next section. Huge variations of $N_{H}$ can be seen, scaling up to a factor of 10 . The unabsorbed $0.2-10 \mathrm{keV}$ luminosity level (outside the eclipse) is compatible with $L_{X} \sim 10^{36} \mathrm{erg} \mathrm{s}^{-1}$, assuming a distance of 8 kpc [6]. Minor variations on the flux can be interpreted as variation of the instantaneous accretion rate $(\dot{M})$ onto the neutron star. Inside the eclipse the flux drops by a factor of $\sim 200$. In the following analysis, we assumed a distance $D \approx 8 \mathrm{kpc}$, a circular orbit ( $e=0)$, and an edge-on geometry $\left(i=90^{\circ}\right)$.

\section{Discussion}

\section{Variability of absorption along the orbit}

We constructed [5] a 3D model of the OB supergiant stellar wind to investigated the behavior of the intrinsic column density, $\mathrm{N}_{\mathrm{H}}$, as a function of phase, and to identify potential structure of the wind during the orbit. We approximated the wind structure with two components, the unperturbed wind $\left(\rho_{\text {wind }}\right)$ and a tail-like hydrodynamic perturbation $\left(\rho_{\text {tail }}\right)$ related to the presence of the neutron star. These shocks are produced by hydrodynamical simulations [2] but produce a $\mathrm{N}_{\mathrm{H}}$ of up to $\sim 10^{22} \mathrm{~cm}^{-2}$, which is too small to account for the variability observed in IGR J17252-3616.

The unperturbed stellar wind was modeled by assuming a standard wind profile [3]

$$
v(r)=v_{\infty}\left(1-\frac{R_{*}}{r}\right)^{\beta}
$$

where $v(r)$ is the wind velocity at distance $r$ from the stellar center, $v_{\infty}$ is the terminal velocity of the wind, and $\beta$ is a parameter describing the wind gradient. The conservation of mass provides the radial density distribution of the stellar wind. The unperturbed stellar wind is a good approximation

\footnotetext{
${ }^{3}$ http://heasarc.gsfc.nasa.gov/docs/xanadu/xspec/

${ }^{4}$ As the $\mathrm{X}$-ray continuum illuminating the gas emitting the Fe fluorescent line cannot be measured during the eclipse, we calculated a corrected $\mathrm{Fe} \mathrm{K} \alpha \mathrm{EW}$ by assuming a constant continuum flux of $1.8 \times 10^{-3} \mathrm{ph} \mathrm{keV}^{-1} \mathrm{~cm}^{-2} \mathrm{~s}^{-1}$
} 

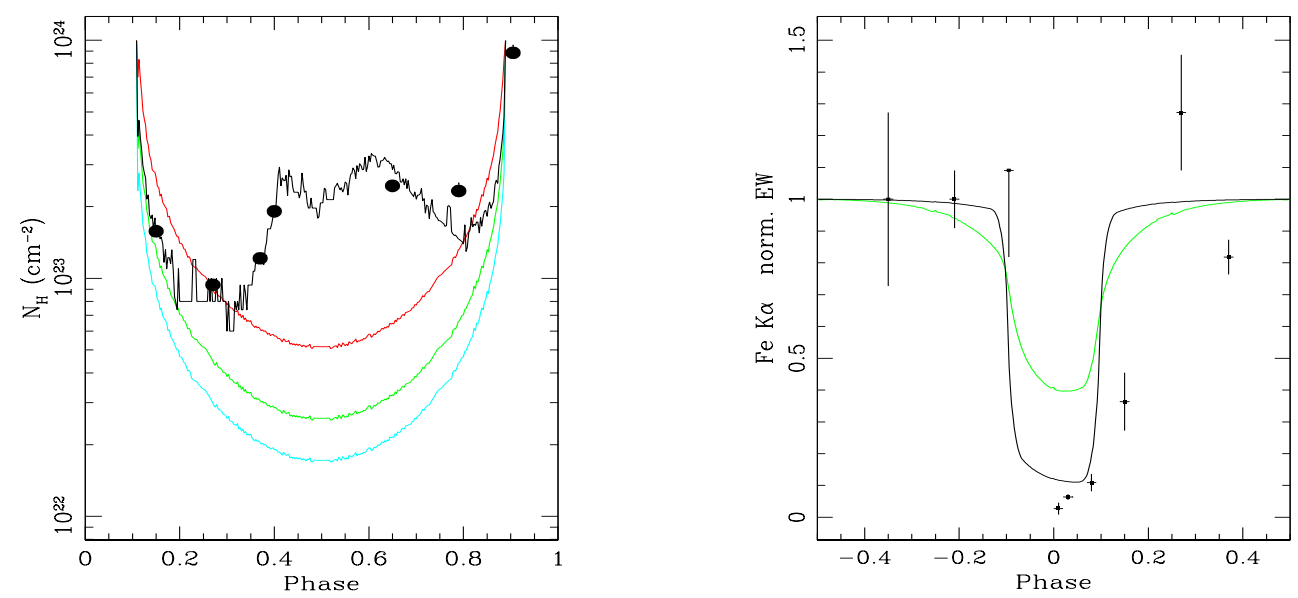

Figure 1: Left panel: Simulated $\mathrm{N}_{H}$ profiles together with the data. Smooth stellar wind is illustrated by cyan, green, and, red curves as a function of $\dot{M} / v_{\infty}=0.7,1,2 \times 10^{-16} \mathrm{M}_{\odot} / \mathrm{km}$, respectively. Black solid line shows the total $\mathrm{N}_{H}$ consisting of the unperturbed stellar wind (green curve) and the tail-like extended component. Right panel: Iron $\mathrm{K} \alpha$ corrected equivalent width during the orbit. Green curve indicated the perdition of the hydrodynamical tail and the black curve the addition of the central cocoon.

within the orbit of the neutron star. Hydrodynamical simulations [2] of HMXB have shown that the wind can be highly disrupted by the neutron star beyond the orbit.

To estimate the terminal velocity of the unperturbed wind, we studied the $\mathrm{N}_{\mathrm{H}}$ variability using three different sets of parameters (Fig. 1 left). The mass-loss rate and terminal velocity are constrained by the data to be in the range $\dot{M}_{w} / v_{\infty} \sim(0.7-2) \times 10^{-16} \mathrm{M}_{\odot} / \mathrm{km}(\beta$ has a very limited impact on the results, so we used 0.7).

The fraction of the wind captured by the neutron star could be estimated from the accretion radius $r_{a c c}=2 G M_{X} /\left(v_{o r b}^{2}+v^{2}\right) \sim 2 \cdot 10^{11} \mathrm{~cm}$ (where $v_{o r b}=250 \mathrm{~km} / \mathrm{s}$ is the orbital velocity) as $f \sim \pi r_{\text {acc }}^{2} / 4 \pi R_{\text {orb }}^{2} \sim 7.5 \cdot 10^{-4}$. The mass-loss rate is therefore $\dot{M}_{w} \sim f^{-1} \dot{M} \approx 1.5 \cdot 10^{-6} \mathrm{M}_{\odot} / \mathrm{yr}$ and the terminal velocity of the wind is constrained to be in the interval $v_{\infty} \sim 250-600 \mathrm{~km} / \mathrm{h}$.

In our simulation, we adopted a terminal velocity $v_{\infty}=400 \mathrm{~km} / \mathrm{sec}$, a stellar radius $R_{*}=29 R_{\odot}$, a wind gradient $\beta=0.7$, and a mass loss rate $\dot{M}_{*}=1.35 \times 10^{-6} \mathrm{M}_{\odot} \mathrm{yr}^{-1}$.

We assumed that the tail-like structure is created very close to the neutron star and opens up with distance. The density of the material inside the 'tail' decreases with distance to ensure mass conservation. Its distribution follows a 'horn'-like shape with a circular section. We adjusted the density of the tail-like structure to match the observations. The density distribution $\rho_{\text {wind }}+\rho_{\text {tail }}$ is displayed in figure 2 left panel. The supergiant is located at the center (black disk). The tail-like structure covers about half of the orbit.

Figure 1 (left panel) displays the simulated $\mathrm{N}_{\mathrm{H}}$ variability from the above density distribution together with the observed data points. The data and the model shows that the tail-like perturbation is essential to understand the observed variations. 


\section{Variability of the $\mathrm{Fe} \mathrm{K} \alpha$ line during the orbit}

Assuming that the intrinsic X-ray flux is unaffected by the eclipse, the $\mathrm{Fe} \mathrm{K} \alpha$ equivalent width drops by a factor $\sim 10$ during the eclipse in an orbital phase interval of $\sim 0.1$. This indicates that the radius of the region emitting $\mathrm{Fe} K \alpha$ is smaller than half of the stellar radius $\left(<10^{12} \mathrm{~cm}\right)$ and far more compact than the tail structure responsible for the absorption variability profile.

Outside the eclipse, the equivalent width of the $\mathrm{Fe} \mathrm{K} \alpha$ line is of the order of $100 \mathrm{eV}$. Following Matt (2002) [7] and assuming a spherical transmission geometry, this corresponds to a column density of $\mathrm{N}_{\mathrm{H}} \sim 2 \cdot 10^{23} \mathrm{~cm}^{-2}$. As this additional absorption is not observed, the region emitting $\mathrm{Fe} \mathrm{K} \alpha$ must be partially ionized.

It is therefore very likely that the dense cocoon corresponds to the inner and ionized region of the hydrodynamical tail. We thus added this partially ionized cocoon in our simulations, using a density of $3 \cdot 10^{11} \mathrm{~cm}^{-3}$ within a radius of $\sim 6 \cdot 10^{11} \mathrm{~cm} \sim 3 \mathrm{R}_{\text {acc. }}$. The Fe $\mathrm{K} \alpha$ emissivity map (fig. 2 right panel) was calculated by applying an illuminating radiation field $\left(\sim 1 / r^{2}\right)$ to the density distribution. Figure 1 right panel displays the resulting simulated profile of the $\mathrm{Fe} \mathrm{K} \alpha$ equivalent width together with the observed data. The green curve shows the variations in the $\mathrm{Fe}$ $\mathrm{K} \alpha$ equivalent width expected from the wind density profile excluding the central cocoon, which obviously could not reproduce the data. The black curve accounts for the dense central cocoon. The exact profile of the eclipse is related to the size and density profile of the cocoon. No effort has been made to obtain an exact match to the data.

The majority of the $\mathrm{Fe} \mathrm{K} \alpha$ is formed in a region that is small enough to allow for pulsation of the Fe $\mathrm{K} \alpha$ line. We searched for such pulsations in our longest and almost uninterrupted observation. Folded lightcurves were built in the energy bands $6.2-6.7 \mathrm{keV}, 2-6.1 \mathrm{keV}$, and $6.8-10$ $\mathrm{keV}$ and resulted in a pulse fraction of $49 \pm 5 \%, 58 \pm 3 \%$, and $57 \pm 4 \%$, respectively. The ratio of the line flux to the continuum in the energy range $6.2-6.7 \mathrm{keV}$ is $\sim 0.25$. Assuming that the line is not pulsed, we infer an $\mathrm{Fe} \mathrm{K} \alpha$ pulse fraction of $\sim 50 \%$, which is in good agreement with the above measurement. Weak Fe $\mathrm{K} \alpha$ pulsation can be explained if the cocoon is isotropic.
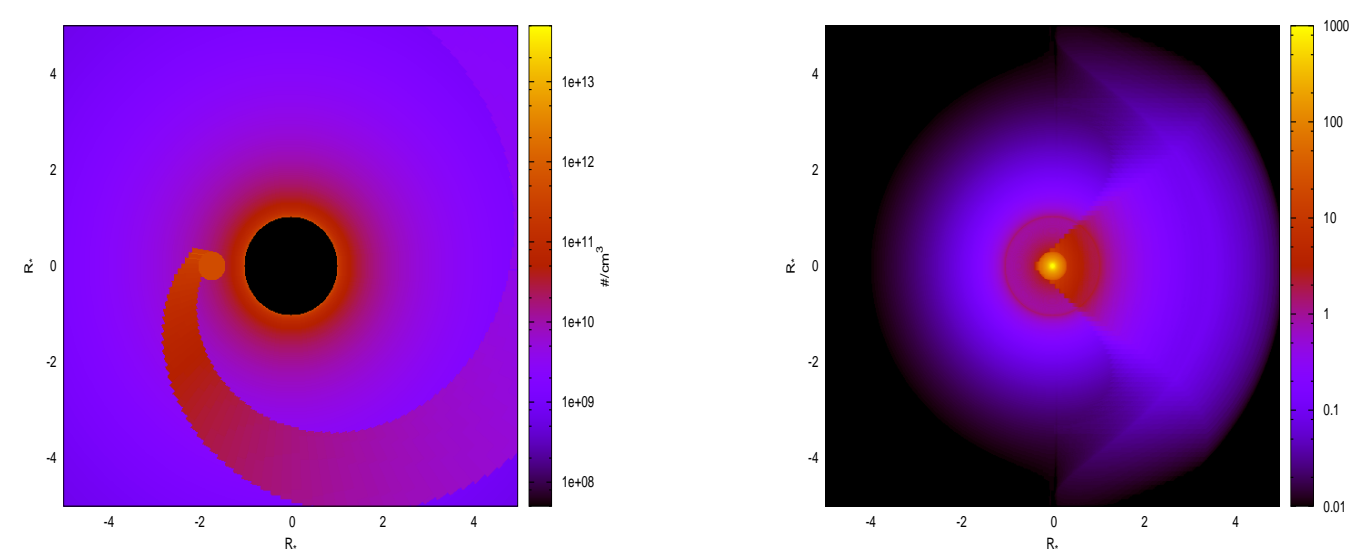

Figure 2: Left panel: Number density distribution in the plane of the orbit including a smooth stellar wind and a tail-like perturbation. The black disk at the center represents the supergiant companion. Right panel: Integrated iron $\mathrm{K} \alpha$ emissivity (relative units) centered on the neutron star at phase $\phi=0.5$. The extended tail can be seen on the right. 


\section{Summary}

The absorbing column density and the $\mathrm{Fe} \mathrm{K} \alpha$ emission line show remarkable variations. The column density, always above $10^{23} \mathrm{~cm}^{-2}$, increases towards $10^{24} \mathrm{~cm}^{-2}$ close to the eclipse, as expected for a spherically symmetric wind. The wind velocity is unusually small close to $v_{\infty}=400$ $\mathrm{km} / \mathrm{s}$. An additional excess of absorption of $2 \cdot 10^{23} \mathrm{~cm}^{-2}$ is observed for orbital phases $\phi>0.3$, which is found to represent a hydrodynamical tail trailing behind the neutron star.

During the eclipse, the equivalent width of the $\mathrm{Fe} \mathrm{K} \alpha$ line drops by a factor $>10$ indicating that most of the line is emitted in a cocoon surrounding the pulsar, with a size of a few accretion radii. This cocoon is ionized and corresponds to the inner region of the hydrodynamical tail

The parameters of the IGR J17252 - 3616 are very similar to these of Vela X-1, except for the smaller wind velocity. We argue that the persistently large absorption column density is related to the hydrodynamical tail, which has been strengthened by the low wind velocity. The tail is a persistent structure dissolving on a timescale comparable to the orbital period.

Our interpretation can be tested using numerical hydrodynamical simulations and high resolution optical/infrared spectroscopy. If confirmed, half of the persistent sgHMXB may have stellar wind speeds several times lower than usually measured.

\section{References}

[1] Anders E. \& Grevesse N. (1989) Geochimica et Cosmochimica Acta 53, 197

[2] Blondin J. M. et al. (1990) ApJ, 356, 591

[3] Castor, J. I., Abbott, D. C., Klein, R. I. (1975) ApJ, 195, 157

[4] Corbet, R.H.D. et al. (2005) ApJ, 633, 377

[5] Manousakis, A. \& Walter, R. (2011) A\&A, 526, 62.

[6] Mason A. B. et al. (2009) A\&A, 505, 281

[7] Matt, G. (2002) MNRAS, 337, 147

[8] Rahoui, F. et al. (2008) A\&A, 484, 801

[9] Takeuchi Y. et al. (1990) PASJ, 42, 287

[10] Tawara Y. et al. (1989) PASJ, 41, 473

[11] Thompson T.W.J. et al (2007) ApJ, 661, 447

[12] Walter, R. et al. (2004) ATel. 229

[13] Walter, R., Zurita Heras J., Bassani, L., et al. 2006 A\& A 453, 133

[14] Walter, R., Rohlfs, R., Meharga, M.T et al. 2010, 8th INTEGRAL workshop, 2010 PoS 162

[15] Warwick, R. S. et al. 1988, MNRAS, 232, 551

[16] White, N.E. 1989, A\& A Rev., 1, 85

[17] Zurita-Heras J.A. et al. (2006) A\&A, 448, 261 